\title{
On Katugampola fractional order derivatives and Darboux problem for differential equations
}

\author{
Djalal Boucenna $^{1}$, Abdellatif Ben Makhlouf ${ }^{2}$ and Mohamed Ali Hammami ${ }^{3}$ \\ 1 Laboratory of Advanced Materials, Faculty of Sciences, \\ Badji Mokhtar-Annaba University, P.O. Box 12, Annaba, 23000, Algeria. \\ 2 Department of Mathematics, College of Science, \\ Jouf University, Aljouf, Saudi Arabia \\ Department of Mathematics, Faculty of Sciences of Sfax, \\ Route Soukra, BP 1171, 3000 Sfax, Tunisia \\ benmakhloufabdellatif@gmail.com \\ 3 Department of Mathematics, Faculty of Sciences of Sfax, \\ Route Soukra, BP 1171, 3000 Sfax, Tunisia
}

\begin{abstract}
In this paper, we investigate the existence and uniqueness of solutions for the Darboux problem of partial differential equations with Caputo-Katugampola fractional derivative.
\end{abstract}

\section{RESUMEN}

En este artículo investigamos la existencia y unicidad de soluciones para el problema de Darboux de ecuaciones diferenciales parciales con derivada fraccional de CaputoKatugampola.

Keywords and Phrases: Darboux problem, Fractional differential equations, Caputo-Katugampola derivative.

2010 AMS Mathematics Subject Classification: 34A34, 34A08, 65L20. 


\section{Introduction}

To investigate many different fields of science and engineering, the fractional calculus represents a powerful tool, with many applications in mathematical physics, hydrology, finance, astrophysics, thermodynamics, statistical mechanics, biophysics, control theory, cosmology, bioengineering and so on, [․ 6 , 6].

In recent years, there has been an important works in ordinary and partial fractional differential equations. For the Caputo fractional-order ordinary differential equations case, see Kilbas et al. [7, Miller and Ross [8]. In addition, Yunru Bai and Hua Kong have treated the existence of solution for nonlinear Caputo-Hadamard fractional differential equations in [9]. For the Caputo fractional-order partial differential equations case, see the work of Tian Liang Guo and KanJian Zhang in [10. Furthermore, Xianmin Zhang has investigated the Caputo-Hadamard partial fractional differential equations in [11. The choice of an appropriate fractional derivative (or integral) depends on the considered system, and for this reason there are a large number of works devoted to different fractional operators.

Recently, U. Katugampola presented new types of fractional operators, which generalize both the Riemann-Liouville and Hadamard fractional operators 4. Although the Katugampola fractional integral operator is an Erdélyi-Kober type operator [13] author in [14 argued that is not possible to obtain Hadamard equivalence operators from Erdélyi-Kober type operators. In this sense, Almeida, Malinowska and Odzijewicz [2] introduced a new fractional operator, called the Caputo-Katugampola derivative, which generalizes the concept of Caputo and Caputo-Hadamard fractional derivatives. It turns out that, the new operator is the left inverse of the Katugampola fractional integral and keeps some of the fundamental properties of the Caputo and CaputoHadamard fractional derivatives. Such derivative is the generalization of the Caputo and CaputoHadamard fractional derivative. The existence and uniqueness of the solution of the ordinary Caputo-Katugampola differential equations is given in [3]. A. Cernea in [12] studied a Darboux problem associated to a fractional hyperbolic integro-differential inclusion defined by CaputoKatugampola fractional derivative and several existence results for this problem are proved.

In this paper, we study the existence and uniqueness of solutions of the following partial differential equation with Caputo-Katugampola fractional derivative

$$
\begin{aligned}
{ }^{C} D_{a^{+}}^{\alpha, \rho} u(x, y)= & f(x, y, u(x, y)),(x, y) \in J=\left[a_{1}, b_{1}\right] \times\left[a_{2}, b_{2}\right], \\
& u\left(x, a_{2}\right)=\varphi(x), x \in\left[a_{1}, b_{1}\right], \\
& u\left(a_{1}, y\right)=\psi(y), y \in\left[a_{2}, b_{2}\right], \\
& \varphi\left(a_{1}\right)=\psi\left(a_{2}\right),
\end{aligned}
$$


where $f: J \times \mathbb{R} \rightarrow \mathbb{R}, \varphi:\left[a_{1}, b_{1}\right] \rightarrow \mathbb{R}$ and $\psi:\left[a_{2}, b_{2}\right] \rightarrow \mathbb{R}$ are given continuous functions.

The rest of the paper is organized as follows. Some definitions and preliminaries are presented in Sect. 2. Finally, the existence and uniqueness results, is given in Sect. 3.

\section{Preliminaries}

In this section, we introduce notations, definitions, and preliminary facts which are used throughout this paper.

Definition 1. [2, 3, 4] Given $\alpha>0, \rho>0$ and an interval $[a, b]$ of $R$, where $0<a<b$. The Katugampola fractional integral of a function $u \in L^{1}([a, b])$ is defined by

$$
I_{a^{+}}^{\alpha, \rho} u(t)=\frac{\rho^{1-\alpha}}{\Gamma(\alpha)} \int_{a}^{t} \frac{s^{\rho-1} u(s)}{\left(t^{\rho}-s^{\rho}\right)^{1-\alpha}} d s,
$$

where $\Gamma$ is the Gamma function.

Definition 2. [2, 3, 4] Given $\alpha>0, \rho>0$ and an interval $[a, b]$ of $R$, where $0<a<b$. The Katugampola fractional derivative is defined by

$$
D_{a^{+}}^{\alpha, \rho} u(t)=\frac{\rho^{\alpha}}{\Gamma(1-\alpha)} t^{1-\rho} \frac{d}{d t} \int_{a}^{t} \frac{s^{\rho-1} u(s)}{\left(t^{\rho}-s^{\rho}\right)^{\alpha}} d s .
$$

Definition 3. [2, 3, 4] Given $0<\alpha<1, \rho>0$ and an interval $[a, b]$ of $R$, where $0<a<b$. The Caputo-Katugampola fractional derivative is defined by

$$
\begin{aligned}
{ }^{C} D_{a^{+}}^{\alpha, \rho} u(t)= & D_{a^{+}}^{\alpha, \rho}[u(t)-u(a)] \\
& =\frac{\rho^{\alpha}}{\Gamma(1-\alpha)} t^{1-\rho} \frac{d}{d t} \int_{a}^{t} \frac{s^{\rho-1}[u(s)-u(a)]}{\left(t^{\rho}-s^{\rho}\right)^{\alpha}} d s .
\end{aligned}
$$

Definition 4. Let $0<a_{i}<b_{i}, i=1,2$ reals numbers, $a=\left(a_{1}, a_{2}\right)$ and $u:\left[a_{1}, b_{1}\right] \times\left[a_{2}, b_{2}\right] \rightarrow \mathbb{R}$ be an integrable function. The mixed Katugampola fractional integrals of order $\alpha=\left(\alpha_{1}, \alpha_{2}\right)$, and parameter $\rho=\left(\rho_{1}, \rho_{2}\right)$ is defined by

$$
I_{a^{+}}^{\alpha, \rho} u(x, y)=\frac{\rho_{1}^{1-\alpha_{1}} \rho_{2}^{1-\alpha_{2}}}{\Gamma\left(\alpha_{1}\right) \Gamma\left(\alpha_{2}\right)} \int_{a_{1}^{+}}^{x} \int_{a_{2}^{+}}^{y} \frac{s^{\rho_{1}-1} t^{\rho_{2}-1}}{\left(x^{\rho_{1}}-s^{\rho_{1}}\right)^{1-\alpha_{1}}\left(y^{\rho_{2}}-t^{\rho_{2}}\right)^{1-\alpha_{2}}} u(s, t) d t d s .
$$

where $\alpha_{1}, \alpha_{2}, \rho_{1}$ and $\rho_{2}$ are strictly positives.

Definition 5. Let $0<a_{i}<b_{i}, i=1,2$ reals numbers, $a=\left(a_{1}, a_{2}\right)$ and $u:\left[a_{1}, b_{1}\right] \times\left[a_{2}, b_{2}\right] \rightarrow \mathbb{R}$ be a function. The mixed Katugampola fractional derivative of order $\alpha=\left(\alpha_{1}, \alpha_{2}\right)$, and parameter 
$\rho=\left(\rho_{1}, \rho_{2}\right)$ is defined by

$$
\begin{aligned}
D_{a^{+}}^{\alpha, \rho} u(x, y)= & x^{1-\rho_{1}} y^{1-\rho_{2}} D_{x, y}^{2} I_{a^{+}}^{1-\alpha, \rho} u(x, y) \\
= & \frac{x^{1-\rho_{1}} y^{1-\rho_{2}} \rho_{1}^{\alpha_{1}} \rho_{2}^{\alpha_{2}}}{\Gamma\left(1-\alpha_{1}\right) \Gamma\left(1-\alpha_{2}\right)} D_{x, y}^{2} \int_{a_{1}^{+}}^{x} \int_{a_{2}^{+}}^{y} \frac{s^{\rho_{1}-1} t^{\rho_{2}-1}}{\left(x^{\rho_{1}}-s^{\rho_{1}}\right)^{\alpha_{1}}\left(y^{\rho_{2}}-t^{\rho_{2}}\right)^{\alpha_{2}}} \\
& \times u(s, t) d t d s .
\end{aligned}
$$

Where $\left(\alpha_{1}, \alpha_{2}\right) \in(0,1)^{2}, D_{x, y}^{2}=\frac{\partial^{2}}{\partial x \partial y}$ and $\rho_{1}, \rho_{2}$ are strictly positives.

Definition 6. Let $0<a_{i}<b_{i}, i=1,2$ reals numbers, $a=\left(a_{1}, a_{2}\right)$ and $u:\left[a_{1}, b_{1}\right] \times\left[a_{2}, b_{2}\right] \rightarrow \mathbb{R}$ be a function. The mixed Caputo-Katugampola fractional derivative of order $\alpha=\left(\alpha_{1}, \alpha_{2}\right)$, and parameter $\rho=\left(\rho_{1}, \rho_{2}\right)$ is defined by

$$
{ }^{C} D_{a^{+}}^{\alpha, \rho} u(x, y)=D_{a^{+}}^{\alpha, \rho}\left(u(x, y)-u\left(x, a_{2}\right)-u\left(a_{1}, y\right)+u\left(a_{1}, a_{2}\right)\right)
$$

where $\left(\alpha_{1}, \alpha_{2}\right) \in(0,1)^{2}$ and $\rho_{1}, \rho_{2}$ are strictly positives.

Lemma 2.1. Let $0<a_{i}<b_{i}, i=1,2$ reals numbers, $a=\left(a_{1}, a_{2}\right)$ and $u:\left[a_{1}, b_{1}\right] \times\left[a_{2}, b_{2}\right] \rightarrow \mathbb{R} i s$ an absolutely continuous function. The mixed Caputo-Katugampola fractional derivative of order $\alpha=\left(\alpha_{1}, \alpha_{2}\right)$, and parameter $\rho=\left(\rho_{1}, \rho_{2}\right)$ is given by

$$
\begin{aligned}
{ }^{C} D_{a^{+}}^{\alpha, \rho} u(x, y) & =I_{a^{+}}^{1-\alpha, \rho}\left(x^{1-\rho_{1}} y^{1-\rho_{2}} D_{x, y}^{2} u(x, y)\right) \\
& =\frac{\rho_{1}^{\alpha_{1}} \rho_{2}^{\alpha_{2}}}{\Gamma\left(1-\alpha_{1}\right) \Gamma\left(1-\alpha_{2}\right)} \int_{a_{1}^{+}}^{x} \int_{a_{2}^{+}}^{y} \frac{D_{s, t}^{2} u(s, t)}{\left(x^{\rho_{1}}-s^{\rho_{1}}\right)^{\alpha_{1}}\left(y^{\rho_{2}}-t^{\rho_{2}}\right)^{\alpha_{2}}} d t d s
\end{aligned}
$$

almost everywhere, where $\left(\alpha_{1}, \alpha_{2}\right) \in(0,1)^{2}, D_{s, t}^{2}=\frac{\partial^{2}}{\partial s \partial t}$ and $\rho_{1}, \rho_{2}$ are strictly positives.

Lemma 2.2. Let $0<a_{i}<b_{i}, i=1,2$ reals numbers, $a=\left(a_{1}, a_{2}\right)$ and $u:\left[a_{1}, b_{1}\right] \times\left[a_{2}, b_{2}\right] \rightarrow \mathbb{R}$ be an integrable function. Then

$$
I_{a^{+}}^{\alpha, \rho} I_{a^{+}}^{\beta, \rho} u(x, y)=I_{a^{+}}^{\alpha+\beta, \rho} u(x, y)
$$

almost everywhere, where $\alpha=\left(\alpha_{1}, \alpha_{2}\right), \beta=\left(\beta_{1}, \beta_{2}\right)$ and parameter $\rho=\left(\rho_{1}, \rho_{2}\right)$. If additionally $u$ is a continuous function, then the identity (2.1) holds everywhere. 
Proof. Using Fubini's Theorem we get

$$
\begin{aligned}
& I_{a^{+}}^{\alpha, \rho} I_{a^{+}}^{\beta, \rho} u(x, y)= \frac{\rho_{1}^{1-\alpha_{1}} \rho_{2}^{1-\alpha_{2}}}{\Gamma\left(\alpha_{1}\right) \Gamma\left(\alpha_{2}\right)} \int_{a_{1}^{+}}^{x} \int_{a_{2}^{+}}^{y} \frac{s_{1}^{\rho_{1}-1} s_{2}^{\rho_{2}-1} I_{a^{+}}^{\beta, \rho} u\left(s_{1}, s_{2}\right)}{\left(x^{\rho_{1}}-s_{1}^{\rho_{1}}\right)^{1-\alpha_{1}}\left(y^{\rho_{2}}-s_{2}^{\rho_{2}}\right)^{1-\alpha_{2}}} d s_{2} d s_{1} \\
&= \frac{\rho_{1}^{1-\beta_{1}} \rho_{2}^{1-\beta_{2}}}{\Gamma\left(\beta_{1}\right) \Gamma\left(\beta_{2}\right)} \frac{\rho_{1}^{1-\alpha_{1}} \rho_{2}^{1-\alpha_{2}}}{\Gamma\left(\alpha_{1}\right) \Gamma\left(\alpha_{2}\right)} \int_{a_{1}^{+}}^{x} \int_{a_{2}^{+}}^{y} \frac{s_{1}^{\rho_{1}-1} s_{2}^{\rho_{2}-1}}{\left(x^{\rho_{1}}-s_{1}^{\rho_{1}}\right)^{1-\alpha_{1}}\left(y^{\rho_{2}}-s_{2}^{\rho_{2}}\right)^{1-\alpha_{2}}} \times \\
& \int_{a_{1}^{+}}^{s_{1}} \int_{a_{2}^{+}}^{s_{2}} \frac{t_{1}^{\rho_{1}-1} t_{2}^{\rho_{2}-1}}{\left(s_{1}^{\rho_{1}}-t_{1}^{\rho_{1}}\right)^{1-\beta_{1}}\left(s_{2}^{\rho_{2}}-t_{2}^{\rho_{2}}\right)^{1-\beta_{2}}} u\left(t_{1}, t_{2}\right) d t_{2} d t_{1} d s_{2} d s_{1} \\
&= \frac{\rho_{1}^{1-\beta_{1}} \rho_{2}^{1-\beta_{2}}}{\Gamma\left(\beta_{1}\right) \Gamma\left(\beta_{2}\right)} \frac{\rho_{1}^{1-\alpha_{1}} \rho_{2}^{1-\alpha_{2}}}{\Gamma\left(\alpha_{1}\right) \Gamma\left(\alpha_{2}\right)} \int_{a_{1}^{+}}^{x} \int_{a_{2}^{+}}^{y} t_{1}^{\rho_{1}-1} t_{2}^{\rho_{2}-1} u\left(t_{1}, t_{2}\right) \times \\
& \int_{t_{1}}^{x} \int_{t_{2}}^{y} \frac{s_{1}^{\rho_{1}-1} s_{2}^{\rho_{2}-1} d s_{2} d s_{1} d t_{2} d t_{1}}{\left(x^{\rho_{1}}-s_{1}^{\rho_{1}}\right)^{1-\alpha_{1}}\left(y^{\rho_{2}}-s_{2}^{\rho_{2}}\right)^{1-\alpha_{2}}\left(s_{1}^{\rho_{1}}-t_{1}^{\rho_{1}}\right)^{1-\beta_{1}}\left(s_{2}^{\rho_{2}}-t_{2}^{\rho_{2}}\right)^{1-\beta_{2}}}
\end{aligned}
$$

Using the change of variables

$$
x=\frac{\left(s_{1}^{\rho_{1}}-t_{1}^{\rho_{1}}\right)^{1-\beta_{1}}}{\left(x^{\rho_{1}}-t_{1}^{\rho_{1}}\right)^{1-\alpha_{1}}} \text { and } y=\frac{\left(s_{2}^{\rho_{2}}-t_{2}^{\rho_{2}}\right)^{1-\beta_{2}}}{\left(y^{\rho_{2}}-t_{2}^{\rho_{2}}\right)^{1-\alpha_{2}}},
$$

we get

$$
\begin{aligned}
& \int_{t_{1}}^{x} \int_{t_{2}}^{y} \frac{s_{1}^{\rho_{1}-1} s_{2}^{\rho_{2}-1}}{\left(x^{\rho_{1}}-s_{1}^{\rho_{1}}\right)^{1-\alpha_{1}}\left(y^{\rho_{2}}-s_{2}^{\rho_{2}}\right)^{1-\alpha_{2}}} \frac{1}{\left(s_{1}^{\rho_{1}}-t_{1}^{\rho_{1}}\right)^{1-\beta_{1}}\left(s_{2}^{\rho_{2}}-t_{2}^{\rho_{2}}\right)^{1-\beta_{2}}} d s_{2} d s_{1} \\
= & \int_{t_{1}}^{x} \frac{s_{1}^{\rho_{1}-1}}{\left(x^{\rho_{1}}-s_{1}^{\rho_{1}}\right)^{1-\alpha_{1}}\left(s_{1}^{\rho_{1}}-t_{1}^{\rho_{1}}\right)^{1-\beta_{1}}} d s_{1} \times \int_{t_{2}}^{y} \frac{s_{2}^{\rho_{2}-1}}{\left(y^{\rho_{2}}-s_{2}^{\rho_{2}}\right)^{1-\alpha_{2}}\left(s_{2}^{\rho_{2}}-t_{2}^{\rho_{2}}\right)^{1-\beta_{2}}} d s_{2} \\
= & \frac{\left(x^{\rho_{1}}-t_{1}^{\rho_{1}}\right)}{\rho_{1}} \frac{\left(y^{\rho_{2}}-t_{2}^{\rho_{2}}\right)}{\rho_{2}} \int_{0}^{1}(1-x)^{\alpha_{1}-1} x^{\beta_{1}} d x \int_{0}^{1}(1-y)^{\alpha_{1}-1} y^{\beta_{1}} d y \\
= & \frac{\left(x^{\rho_{1}}-t_{1}^{\rho_{1}}\right)}{\rho_{1}} \frac{\left(y^{\rho_{2}}-t_{2}^{\rho_{2}}\right)}{\rho_{2}} B\left(\alpha_{1}, \beta_{1}\right) B\left(\alpha_{2}, \beta_{2}\right) \\
= & \frac{\left(x^{\rho_{1}}-t_{1}^{\rho_{1}}\right)}{\rho_{1}} \frac{\left(y^{\rho_{2}}-t_{2}^{\rho_{2}}\right)}{\rho_{2}} \frac{\Gamma\left(\alpha_{1}\right) \Gamma\left(\beta_{1}\right)}{\Gamma\left(\alpha_{1}+\beta_{1}\right)} \frac{\Gamma\left(\alpha_{2}\right) \Gamma\left(\beta_{2}\right)}{\Gamma\left(\alpha_{2}+\beta_{2}\right)} .
\end{aligned}
$$

From (2.2) and (2.3) we obtain (2.1).

Lemma 2.3. Let $0<a_{i}<b_{i}, i=1,2$ reals numbers, $a=\left(a_{1}, a_{2}\right)$ and $u:\left[a_{1}, b_{1}\right] \times\left[a_{2}, b_{2}\right] \rightarrow \mathbb{R}$ be an integrable function. Then

$$
D_{a^{+}}^{\alpha, \rho} I_{a^{+}}^{\alpha, \rho} u(x, y)=u(x, y)
$$

almost everywhere, where $\alpha=\left(\alpha_{1}, \alpha_{2}\right) \in(0,1)^{2}$ and parameter $\rho=\left(\rho_{1}, \rho_{2}\right)$.

Proof. From Lemma (2.2), we get

$$
\begin{aligned}
D_{a^{+}}^{\alpha, \rho} I_{a^{+}}^{\alpha, \rho} u(x, y) & =x^{1-\rho_{1}} y^{1-\rho_{2}} D_{x, y}^{2} I_{a^{+}}^{1-\alpha, \rho} I_{a^{+}}^{\alpha, \rho} u(x, y) \\
& =x^{1-\rho_{1}} y^{1-\rho_{2}} D_{x, y}^{2} I_{a^{+}}^{1, \rho} u(x, y) \\
& =u(x, y)
\end{aligned}
$$




\section{$3 \quad$ Existence and uniqueness results}

For the existence and uniqueness of solutions for the problem (1.1)-(1.2) we need the following lemma.

Lemma 3.1. The function $u \in C(J)$ is a solution of fractional order problem (1.1)-(1.2) if and only if

$$
u(x, y)=\varphi(x)+\psi(y)-\varphi\left(a_{1}\right)+I_{a^{+}}^{\alpha, \rho} f(x, y, u(x, y))
$$

Proof. First suppose that $u$ is a solution of the integral equation (3.1). Applied ${ }^{C} D_{a^{+}}^{\alpha, \rho}$ and using Lemma 2.3 we obtain that $u$ solves the the equation (1.1). Since the integral is zero when $x=a_{1}$, or $y=a_{2}$, then the initial conditions in (1.2) are satisfied. Hence $u$ solves the problem (1.1)-(1.2). Conversly, if $u$ is a solution of the problem (1.1)-(1.2). Let

$$
\begin{aligned}
h(x, y) & =f(x, y, u(x, y)) \\
& =D_{a^{+}}^{\alpha, \rho}\left(u(x, y)-u\left(x, a_{2}\right)-u\left(a_{1}, y\right)+u\left(a_{1}, a_{2}\right)\right) \\
& =x^{1-\rho_{1}} y^{1-\rho_{2}} D_{x, y}^{2} I_{a^{+}}^{1-\alpha, \rho}\left[u(x, y)-u\left(x, a_{2}\right)-u\left(a_{1}, y\right)+u\left(a_{1}, a_{2}\right)\right] .
\end{aligned}
$$

Applying the operator $I_{a^{+}}^{1, \rho}$ to (3.2), we get

$$
I_{a^{+}}^{1, \rho} h(x, y)=I_{a^{+}}^{1-\alpha, \rho}\left[u(x, y)-u\left(x, a_{2}\right)-u\left(a_{1}, y\right)+u\left(a_{1}, a_{2}\right)\right] .
$$

Applying the operator $D_{a^{+}}^{1-\alpha, \rho}$ to this equation we find

$$
\begin{aligned}
{\left[u(x, y)-u\left(x, a_{2}\right)-u\left(a_{1}, y\right)+u\left(a_{1}, a_{2}\right)\right] } & =D_{a^{+}}^{1-\alpha, \rho} I_{a^{+}}^{1, \rho} h(x, y) \\
& =\left(x^{1-\rho_{1}} y^{1-\rho_{2}}\right) D_{x, y}^{2} I_{a^{+}}^{\alpha, \rho} I_{a^{+}}^{1, \rho} h(x, y) \\
& =I_{a^{+}}^{\alpha, \rho} h(x, y) .
\end{aligned}
$$

Hence, the proof is complete.

\subsection{Existence of solutions}

In this subsection we study the existence of solutions for the problem (1.1)-(1.2).

Theorem 3.1. Let $k>0, h_{1}^{*}>a_{1}$ and $h_{2}^{*}>a_{2}$.

Define

$$
\begin{gathered}
G=\left\{(x, y, u):(x, y) \in\left[a_{1}, h_{1}^{*}\right] \times\left[a_{2}, h_{2}^{*}\right],\left|u-\varphi(x)-\psi(y)+\varphi\left(a_{1}\right)\right| \leq k\right\}, \\
M=\sup _{(x, y, u) \in G}|f(x, y, u)|
\end{gathered}
$$


and

$$
\left(h_{1}, h_{2}\right)= \begin{cases}\left(h_{1}^{*}, h_{2}^{*}\right) & \text { if } M=0, \\ \left(\min \left(h_{1}^{*},\left(\frac{k^{\frac{1}{2}} \rho_{1}^{\alpha_{1}} \Gamma\left(\alpha_{1}+1\right)}{M^{\frac{1}{2}}}\right)^{\frac{1}{\alpha_{1}}}\right), \min \left(h_{2}^{*},\left(\frac{k^{\frac{1}{2}} \rho_{2}^{\alpha_{2}} \Gamma\left(\alpha_{2}+1\right)}{M^{\frac{1}{2}}}\right)^{\frac{1}{\alpha_{2}}}\right)\right) & \text { otherwise. }\end{cases}
$$

Then, there exists a function $u \in C\left[a_{1}, h_{1}\right] \times\left[a_{2}, h_{2}\right]$ that solves the problem (1.1)-(1.2).

Proof. If $M=0$ then $f(x, y, u)=0$, for all $(x, y, u) \in G$. In this case it is clear that the function $u:\left[a_{1}, h_{1}\right] \times\left[a_{2}, h_{2}\right] \rightarrow \mathbb{R}$ with $u(x, y)=\varphi(x)+\psi(y)-\varphi\left(a_{1}\right)$ is a solution of the problem (1.1)(1.2).

For $M \neq 0$, using Lemma 3.1 we obtain that the problem (1.1)-(1.2) is equivalent to the Volterra integral equation (3.1).

Define the function $T$ by

$$
T(x, y)=\varphi(x)+\psi(y)-\varphi\left(a_{1}\right) .
$$

and the set $U$ by

$$
U=\left\{u \in C\left(\left[a_{1}, h_{1}\right] \times\left[a_{2}, h_{2}\right]\right),\|u-T\|_{\infty} \leq k\right\} .
$$

The set $U$ is nonempty since $T \in U$. It is clear that $U$ is a closed and convex subset of the Banach space of all continuous functions on $\left[a_{1}, h_{1}\right] \times\left[a_{2}, h_{2}\right]$.

We define the operator $A$ on this set $U$ by

$$
(A u)(x, y)=T(x, y)+\frac{\rho_{1}^{1-\alpha_{1}} \rho_{2}^{1-\alpha_{2}}}{\Gamma\left(\alpha_{1}\right) \Gamma\left(\alpha_{2}\right)} \int_{a_{1}^{+}}^{x} \int_{a_{2}^{+}}^{y} \frac{s^{\rho_{1}-1} t^{\rho_{2}-1} f(s, t, u(s, t))}{\left(x^{\rho_{1}}-s^{\rho_{1}}\right)^{1-\alpha_{1}}\left(y^{\rho_{2}}-t^{\rho_{2}}\right)^{1-\alpha_{2}}} d t d s .
$$

We have to show that $A$ has a fixed point. This is done through the Schauder's Fixed Point Theorem.

It is easy to see that $A$ is continuous. Now we show that $A$ is defined to $U$ into itself, let $u \in U$ and $(x, y) \in\left[a_{1}, h_{1}\right] \times\left[a_{2}, h_{2}\right]$ then

$$
\begin{aligned}
|(A u)(x, y)-T(x, y)| & =\frac{\rho_{1}^{1-\alpha_{1}} \rho_{2}^{1-\alpha_{2}}}{\Gamma\left(\alpha_{1}\right) \Gamma\left(\alpha_{2}\right)} \int_{a_{1}^{+}}^{x} \int_{a_{2}^{+}}^{y} \frac{s^{\rho_{1}-1} t^{\rho_{2}-1}|f(s, t, u(s, t))|}{\left(x^{\rho_{1}}-s^{\rho_{1}}\right)^{1-\alpha_{1}}\left(y^{\rho_{2}}-t^{\rho_{2}}\right)^{1-\alpha_{2}}} d t d s \\
& \leq \frac{M \rho_{1}^{1-\alpha_{1}} \rho_{2}^{1-\alpha_{2}}}{\Gamma\left(\alpha_{1}\right) \Gamma\left(\alpha_{2}\right)} \int_{a_{1}^{+}}^{x} \int_{a_{2}^{+}}^{y} \frac{s^{\rho_{1}-1} t^{\rho_{2}-1}}{\left(x^{\rho_{1}}-s^{\rho_{1}}\right)^{1-\alpha_{1}}\left(y^{\rho_{2}}-t^{\rho_{2}}\right)^{1-\alpha_{2}}} d t d s \\
& \leq \frac{M}{\Gamma\left(\alpha_{1}+1\right) \Gamma\left(\alpha_{2}+1\right)}\left(\frac{x^{\rho_{1}}-a_{1}^{\rho_{1}}}{\rho_{1}}\right)^{\alpha_{1}}\left(\frac{y^{\rho_{2}}-a_{2}^{\rho_{2}}}{\rho_{2}}\right)^{\alpha_{2}} \\
& \leq \frac{M}{\rho_{1}^{\alpha_{1}} \rho_{2}^{\alpha_{2}} \Gamma\left(\alpha_{1}+1\right) \Gamma\left(\alpha_{2}+1\right)} h_{1}^{\rho_{1} \alpha_{1}} h_{2}^{\rho_{2} \alpha_{2}} \\
& \leq \frac{M}{\rho_{1}^{\alpha_{1}} \rho_{2}^{\alpha_{2}} \Gamma\left(\alpha_{1}+1\right) \Gamma\left(\alpha_{2}+1\right)} h_{1}^{\alpha_{1}} h_{2}^{\alpha_{2}} \\
& \leq \frac{M}{\rho_{1}^{\alpha_{1}} \rho_{2}^{\alpha_{2}} \Gamma\left(\alpha_{1}+1\right) \Gamma\left(\alpha_{2}+1\right)} \frac{k \rho_{1}^{\alpha_{1}} \rho_{2}^{\alpha_{2}} \Gamma\left(\alpha_{1}+1\right) \Gamma\left(\alpha_{2}+1\right)}{M} \\
& \leq k .
\end{aligned}
$$


Thus, we have $A u \in U$ if $u \in U$. We will now show that $A U=\{A u: u \in U\}$ is relatively compact. This is done by the using Arzela-Ascoli Theorem.

Firstly, we show that $A(U)$ is uniformly bounded. Indeed, let $u \in U$ and $(x, y) \in\left[a_{1}, h_{1}\right] \times\left[a_{2}, h_{2}\right]$ and from the previous step we get

$$
\|A u\|_{\infty} \leq\|T\|_{\infty}+k
$$

Secondly, we show that $A(U)$ is equicontinuous. Indeed, let $\left(x_{1}, y_{1}\right) \in\left[a_{1}, h_{1}\right] \times\left[a_{2}, h_{2}\right],\left(x_{2}, y_{2}\right) \in$ $\left[a_{1}, h_{1}\right] \times\left[a_{2}, h_{2}\right]$ such that $x_{1}<x_{2}$ and $y_{1}<y_{2}$, we have

$$
\begin{aligned}
& \left|(A u)\left(x_{1}, y_{1}\right)-(A u)\left(x_{2}, y_{2}\right)\right| \\
\leq & \left|T\left(x_{1}, y_{1}\right)-T\left(x_{2}, y_{2}\right)\right|+\frac{M \rho_{1}^{1-\alpha_{1}} \rho_{2}^{1-\alpha_{2}}}{\Gamma\left(\alpha_{1}\right) \Gamma\left(\alpha_{2}\right)} \int_{a_{1}^{+}}^{x_{1}} \int_{a_{2}^{+}}^{y_{1}} \frac{s^{\rho_{1}-1} t^{\rho_{2}-1}}{\left(x_{1}^{\rho_{1}}-s^{\rho_{1}}\right)^{1-\alpha_{1}}\left(y_{1}^{\rho_{2}}-t^{\rho_{2}}\right)^{1-\alpha_{2}}} \\
& -\frac{s^{\rho_{1}-1} t^{\rho_{2}-1}}{\left(x_{2}^{\rho_{1}}-s^{\rho_{1}}\right)^{1-\alpha_{1}}\left(y_{2}^{\rho_{2}}-t^{\rho_{2}}\right)^{1-\alpha_{2}}} d t d s \\
& +\frac{M \rho_{1}^{1-\alpha_{1}} \rho_{2}^{1-\alpha_{2}}}{\Gamma\left(\alpha_{1}\right) \Gamma\left(\alpha_{2}\right)} \int_{a_{1}^{+}}^{x_{1}} \int_{y_{1}}^{y_{2}} \frac{s^{\rho_{1}-1} t^{\rho_{2}-1}}{\left(x_{2}^{\rho_{1}}-s^{\rho_{1}}\right)^{1-\alpha_{1}}\left(y_{2}^{\rho_{2}}-t^{\rho_{2}}\right)^{1-\alpha_{2}}} d t d s \\
& +\frac{M \rho_{1}^{1-\alpha_{1}} \rho_{2}^{1-\alpha_{2}}}{\Gamma\left(\alpha_{1}\right) \Gamma\left(\alpha_{2}\right)} \int_{x_{1}}^{x_{2}} \int_{a_{2}^{+}}^{y_{1}} \frac{s^{\rho_{1}-1} t^{\rho_{2}-1}}{\left(x_{2}^{\rho_{1}}-s^{\rho_{1}}\right)^{1-\alpha_{1}}\left(y_{2}^{\rho_{2}}-t^{\rho_{2}}\right)^{1-\alpha_{2}}} d t d s \\
& +\frac{M \rho_{1}^{1-\alpha_{1}} \rho_{2}^{1-\alpha_{2}}}{\Gamma\left(\alpha_{1}\right) \Gamma\left(\alpha_{2}\right)} \int_{x_{1}}^{x_{2}} \int_{y_{1}}^{y_{2}} \frac{s^{\rho_{2}-1}}{\left(x_{2}^{\rho_{1}}-s^{\rho_{1}}\right)^{1-\alpha_{1}}\left(y_{2}^{\rho_{2}}-t^{\rho_{2}}\right)^{1-\alpha_{2}}} d t d s \\
\leq \quad & \left|T\left(x_{1}, y_{1}\right)-T\left(x_{2}, y_{2}\right)\right| \\
& +\frac{3 M}{\rho_{1}^{\alpha_{1}} \rho_{2}^{\alpha_{2}} \Gamma\left(\alpha_{1}\right) \Gamma\left(\alpha_{2}\right)}\left[\left(x_{2}^{\rho_{1}}-a_{1}^{\rho_{1}}\right)^{\alpha_{1}}\left(y_{2}^{\rho_{2}}-y_{1}^{\rho_{2}}\right)^{\alpha_{2}}+\left(y_{2}^{\rho_{2}}-a_{2}^{\rho_{2}}\right)^{\alpha_{2}}\left(x_{2}^{\rho_{1}}-x_{1}^{\rho_{1}}\right)^{\alpha_{1}}\right]
\end{aligned}
$$

Hence, $A(U)$ is equicontinous, since $T$ is uniformly continuous in $\left[a_{1}, h_{1}\right] \times\left[a_{2}, h_{2}\right]$. As a consequence of the Schauder's Fixed Point Theorem, we deduce that $A$ has a fixed point $u$ in $U$. This fixed point is the required solution of the problem (1.1)-(1.2). Hence, the proof is complete.

\subsection{Uniqueness of solutions}

In this subsection we discuss the uniqueness results for the problem (1.1)-(1.2).

Let $u_{1}, u_{2} \in C\left(\left[a_{1}, h_{1}\right] \times\left[a_{2}, h_{2}\right]\right)$, and $(x, y) \in\left[a_{1}, h_{1}\right] \times\left[a_{2}, h_{2}\right]$.

Suppose there exists a constant $L>0$ independent of $x, y, u_{1}$, and $u_{2}$ such that

$$
\left|f\left(x, y, u_{1}\right)-f\left(x, y, u_{2}\right)\right| \leq L\left|u_{1}-u_{2}\right|
$$

then we have

$$
\left\|\left(A u_{1}\right)-\left(A u_{2}\right)\right\|_{C\left(\left[a_{1}, x\right] \times\left[a_{2}, y\right]\right)} \leq \frac{L\left\|u_{1}-u_{2}\right\|_{C\left(\left[a_{1}, x\right] \times\left[a_{2}, y\right]\right)}}{\Gamma\left(\alpha_{1}+1\right) \Gamma\left(\alpha_{2}+1\right)}\left(\frac{x^{\rho_{1}}}{\rho_{1}}\right)^{\alpha_{1}}\left(\frac{y^{\rho_{2}}}{\rho_{2}}\right)^{\alpha_{2}} .
$$


Indeed, let $u_{1}, u_{2} \in C\left(\left[a_{1}, h_{1}\right] \times\left[a_{2}, h_{2}\right]\right),(x, y) \in\left[a_{1}, h_{1}\right] \times\left[a_{2}, h_{2}\right]$ and $(v, w) \in\left[a_{1}, x\right] \times\left[a_{2}, y\right]$, we have

$$
\begin{aligned}
& \left|\left(A u_{1}\right)(v, w)-\left(A u_{2}\right)(v, w)\right| \\
= & \frac{\rho_{1}^{1-\alpha_{1}} \rho_{2}^{1-\alpha_{2}}}{\Gamma\left(\alpha_{1}\right) \Gamma\left(\alpha_{2}\right)} \int_{a_{1}^{+}}^{v} \int_{a_{2}^{+}}^{w} \frac{s^{\rho_{1}-1} t^{\rho_{2}-1}\left|f\left(s, t, u_{1}(s, t)\right)-f\left(s, t, u_{2}(s, t)\right)\right|}{\left(v^{\rho_{1}}-s^{\rho_{1}}\right)^{1-\alpha_{1}}\left(w^{\rho_{2}}-t^{\rho_{2}}\right)^{1-\alpha_{2}}} d t d s \\
\leq & \frac{L \rho_{1}^{1-\alpha_{1}} \rho_{2}^{1-\alpha_{2}}}{\Gamma\left(\alpha_{1}\right) \Gamma\left(\alpha_{2}\right)} \int_{a_{1}^{+}}^{v} \int_{a_{2}^{+}}^{w} \frac{s^{\rho_{1}-1} t^{\rho_{2}-1}}{\left(v^{\rho_{1}}-s^{\rho_{1}}\right)^{1-\alpha_{1}}\left(w^{\rho_{2}}-t^{\rho_{2}}\right)^{1-\alpha_{2}}}\left|u_{1}(s, t)-u_{2}(s, t)\right| d t d s \\
\leq & \frac{L \rho_{1}^{1-\alpha_{1}} \rho_{2}^{1-\alpha_{2}}}{\Gamma\left(\alpha_{1}\right) \Gamma\left(\alpha_{2}\right)}\left\|u_{1}-u_{2}\right\|_{C\left(\left[a_{1}, x\right] \times\left[a_{2}, y\right]\right)} \int_{a_{1}^{+}}^{v} \int_{a_{2}^{+}}^{w} \frac{s^{\rho_{1}-1} t^{\rho_{2}-1}}{\left(v^{\rho_{1}}-s^{\rho_{1}}\right)^{1-\alpha_{1}}\left(w^{\rho_{2}}-t^{\rho_{2}}\right)^{1-\alpha_{2}}} d t d s \\
\leq & \frac{L}{\Gamma\left(\alpha_{1}+1\right) \Gamma\left(\alpha_{2}+1\right)}\left\|u_{1}-u_{2}\right\|_{C\left(\left[a_{1}, x\right] \times\left[a_{2}, y\right]\right)}\left(\frac{v^{\rho_{1}}}{\rho_{1}}\right)^{\alpha_{1}}\left(\frac{w^{\rho_{2}}}{\rho_{2}}\right)^{\alpha_{2}} \\
\leq & \frac{L}{\Gamma\left(\alpha_{1}+1\right) \Gamma\left(\alpha_{2}+1\right)}\left\|u_{1}-u_{2}\right\|_{C\left(\left[a_{1}, x\right] \times\left[a_{2}, y\right]\right)}\left(\frac{x^{\rho_{1}}}{\rho_{1}}\right)^{\alpha_{1}}\left(\frac{y^{\rho_{2}}}{\rho_{2}}\right)^{\alpha_{2}} .
\end{aligned}
$$

From the above inequality we get (3.7).

$$
\left\|\left(A u_{1}\right)-\left(A u_{2}\right)\right\|_{C\left(\left[a_{1}, x\right] \times\left[a_{2}, y\right]\right)} \leq \frac{L\left\|u_{1}-u_{2}\right\|_{C\left(\left[a_{1}, x\right] \times\left[a_{2}, y\right]\right)}}{\Gamma\left(\alpha_{1}+1\right) \Gamma\left(\alpha_{2}+1\right)}\left(\frac{x^{\rho_{1}}}{\rho_{1}}\right)^{\alpha_{1}}\left(\frac{y^{\rho_{2}}}{\rho_{2}}\right)^{\alpha_{2}} .
$$

Next, we have the following result

Theorem 3.2. Suppose that the assumptions of Theorem 3.1 are satisfied. Also let $j \in \mathbb{N},(x, y) \in$ $\left[a_{1}, h_{1}\right] \times\left[a_{2}, h_{2}\right]$ and $u_{1}, u_{2} \in U$. Suppose $f$ satisfies the Lipschitz condition with respect to the third variable with the Lipschitz constant $L$. Then

$$
\left\|A^{j} u_{1}-A^{j} u_{2}\right\|_{C\left(\left[a_{1}, x\right] \times\left[a_{2}, y\right]\right)} \leq \frac{\left(\frac{x^{\rho_{1}}}{\rho_{1}}\right)^{\alpha_{1} j}\left(\frac{y^{\rho_{2}}}{\rho_{2}}\right)^{\alpha_{2} j}}{\Gamma\left(1+\alpha_{1} j\right) \Gamma\left(1+\alpha_{2} j\right)}\left\|u_{1}-u_{2}\right\|_{C\left(\left[a_{1}, x\right] \times\left[a_{2}, y\right]\right)} .
$$

Proof. We will prove (3.8) by induction. In the case $j=0$, the inequality holds. Assume (3.8) is 
true for $j-1 \in \mathbb{N}_{0}$ then for all $(x, y) \in\left[a_{1}, h_{1}\right] \times\left[a_{2}, h_{2}\right]$ and $(v, w) \in\left[a_{1}, x\right] \times\left[a_{2}, y\right]$ we have

$$
\begin{aligned}
& \left|\left(A^{j} u_{1}\right)(v, w)-\left(A^{j} u_{2}\right)(v, w)\right| \\
& =\left|\left(A A^{j-1} u_{1}\right)(v, w)-\left(A A^{j-1} u_{2}\right)(v, w)\right| \\
& =\frac{\rho_{1}^{1-\alpha_{1}} \rho_{2}^{1-\alpha_{2}}}{\Gamma\left(\alpha_{1}\right) \Gamma\left(\alpha_{2}\right)} \int_{a_{1}^{+}}^{v} \int_{a_{2}^{+}}^{w} \frac{s^{\rho_{1}-1} t^{\rho_{2}-1}\left|f\left(s, t, A^{j-1} u_{1}(s, t)\right)-f\left(s, t, A^{j-1} u_{2}(s, t)\right)\right|}{\left(v^{\rho_{1}}-s^{\rho_{1}}\right)^{1-\alpha_{1}}\left(w^{\rho_{2}}-t^{\rho_{2}}\right)^{1-\alpha_{2}}} d t d s \\
& \leq \frac{L \rho_{1}^{1-\alpha_{1}} \rho_{2}^{1-\alpha_{2}}}{\Gamma\left(\alpha_{1}\right) \Gamma\left(\alpha_{2}\right)} \int_{a_{1}^{+}}^{v} \int_{a_{2}^{+}}^{w} \frac{s^{\rho_{1}-1} t^{\rho_{2}-1}\left|A^{j-1} u_{1}(s, t)-A^{j-1} u_{2}(s, t)\right|}{\left(v^{\rho_{1}}-s^{\rho_{1}}\right)^{1-\alpha_{1}}\left(w^{\rho_{2}}-t^{\rho_{2}}\right)^{1-\alpha_{2}}} d t d s \\
& \leq \frac{L \rho_{1}^{1-\alpha_{1}} \rho_{2}^{1-\alpha_{2}}}{\Gamma\left(\alpha_{1}\right) \Gamma\left(\alpha_{2}\right)} \int_{a_{1}^{+}}^{v} \int_{a_{2}^{+}}^{w} \frac{s^{\rho_{1}-1} t^{\rho_{2}-1}\left\|A^{j-1} u_{1}-A^{j-1} u_{2}\right\|_{C\left(\left[a_{1}, s\right] \times\left[a_{2}, t\right]\right)}}{\left(v^{\rho_{1}}-s^{\rho_{1}}\right)^{1-\alpha_{1}}\left(w^{\rho_{2}}-t^{\rho_{2}}\right)^{1-\alpha_{2}}} d t d s \\
& \leq \frac{L^{j} \rho_{1}^{1-\alpha_{1} j} \rho_{2}^{1-\alpha_{2} j}}{\Gamma\left(\alpha_{1}\right) \Gamma\left(\alpha_{2}\right) \Gamma\left(1+\alpha_{1}(j-1)\right) \Gamma\left(1+\alpha_{2}(j-1)\right)}\left\|u_{1}-u_{2}\right\|_{C\left(\left[a_{1}, x\right] \times\left[a_{2}, y\right]\right)} \\
& \int_{a_{1}^{+}}^{v} \int_{a_{2}^{+}}^{w} \frac{s^{\rho_{1}+\alpha_{1} \rho_{1}(j-1)-1} t^{\rho_{2}+\alpha_{2} \rho_{2}(j-1)-1}}{\left(v^{\rho_{1}}-s^{\rho_{1}}\right)^{1-\alpha_{1}}\left(w^{\rho_{2}}-t^{\rho_{2}}\right)^{1-\alpha_{2}}} d t d s \\
& \leq \frac{L^{j} \rho_{1}^{1-\alpha_{1} j} \rho_{2}^{1-\alpha_{2} j}}{\Gamma\left(\alpha_{1}\right) \Gamma\left(\alpha_{2}\right) \Gamma\left(1+\alpha_{1}(j-1)\right) \Gamma\left(1+\alpha_{2}(j-1)\right)}\left\|u_{1}-u_{2}\right\|_{C\left(\left[a_{1}, x\right] \times\left[a_{2}, y\right]\right)} \\
& \times \frac{\Gamma\left(\alpha_{1}\right) \Gamma\left(\alpha_{2}\right) \Gamma\left(1+\alpha_{1}(j-1)\right) \Gamma\left(1+\alpha_{2}(j-1)\right)}{\Gamma\left(1+\alpha_{1} j\right) \Gamma\left(1+\alpha_{2} j\right)} \frac{x^{\rho_{1} \alpha_{1} j}}{\rho_{1}} \frac{y^{\rho_{2} \alpha_{2} j}}{\rho_{2}} \\
& \leq \frac{\left(\frac{x^{\rho_{1}}}{\rho_{1}}\right)^{\alpha_{1} j}\left(\frac{y^{\rho_{2}}}{\rho_{2}}\right)^{\alpha_{2} j}}{\Gamma\left(1+\alpha_{1} j\right) \Gamma\left(1+\alpha_{2} j\right)}\left\|u_{1}-u_{2}\right\|_{C\left(\left[a_{1}, x\right] \times\left[a_{2}, y\right]\right)} \text {. }
\end{aligned}
$$

Hence, the proof is complete.

Theorem 3.3. Let $k, h_{1}^{*}$ and $h_{1}^{*}$ are positive numbers, define the set $G$ as in Theorem 3.1 and assume that the function $f: G \rightarrow \mathbb{R}$ satisfies a Lipschitz condition with respect to the third variable with the Lipschitz constant $L$. Then, there exists a unique solution $u \in C\left(\left[a_{1}, h_{1}\right] \times\left[a_{2}, h_{2}\right]\right)$ for the problem (1.1)-(1.2). Where $h_{1}, h_{2}$ are the same as in Theorem 3.1.

Proof. According to Theorem 3.1 the problem (1.1)-(1.2) has a solution. To prove the uniqueness, we adopt Theorem 3.2 , we use the operato $A$ as defined in (3.5), the function $T$ as defined in (3.3) and the set $U$ as defined in (3.4). We will apply Weissinger's Fixed Point Theorem to prove that $A$ has a unique fixed point.

Let $j \in \mathbb{N}$ and $u_{1}, u_{2} \in C\left(\left[a_{1}, h_{1}\right] \times\left[a_{2}, h_{2}\right]\right)$. From (3.8) and taking the norms on $\left[a_{1}, h_{1}\right] \times\left[a_{2}, h_{2}\right]$, we get

$$
\left\|A^{j-1} u_{1}-A^{j-1} u_{2}\right\|_{C\left(\left[a_{1}, h_{1}\right] \times\left[a_{2}, h_{2}\right]\right)} \leq \frac{\left(\frac{x^{\rho_{1}}}{\rho_{1}}\right)^{\alpha_{1} j}\left(\frac{y^{\rho_{2}}}{\rho_{2}}\right)^{\alpha_{2} j}}{\Gamma\left(1+\alpha_{1} j\right) \Gamma\left(1+\alpha_{2} j\right)}\left\|u_{1}-u_{2}\right\|_{C\left(\left[a_{1}, h_{1}\right] \times\left[a_{2}, h_{2}\right]\right)} .
$$


Let $\omega_{j}=\frac{\left(\frac{x^{\rho_{1}}}{\rho_{1}}\right)^{\alpha_{1} j}\left(\frac{y^{\rho_{2}}}{\rho_{2}}\right)^{\alpha_{2} j}}{\Gamma\left(1+\alpha_{1} j\right) \Gamma\left(1+\alpha_{2} j\right)}$. It is clear that

$$
\sum_{j=0}^{\infty} \omega_{j}=\sum_{j=0}^{\infty} \frac{\left(\left(\frac{x^{\rho_{1}}}{\rho_{1}}\right)^{\alpha_{1}}\left(\frac{y^{\rho_{2}}}{\rho_{2}}\right)^{\alpha_{2}}\right)^{j}}{\Gamma\left(1+\alpha_{1} j\right) \Gamma\left(1+\alpha_{2} j\right)}=\mathbb{E}\left(\left(\alpha_{i}, 1\right)_{i=1,2} ;\left(\left(\frac{x^{\rho_{1}}}{\rho_{1}}\right)^{\alpha_{1}}\left(\frac{y^{\rho_{2}}}{\rho_{2}}\right)^{\alpha_{2}}\right)\right),
$$

hence the series converges. This completes the proof.

\section{Conclusion}

Here we have studied the existence and uniqueness of the solutions for the Darboux problem of partial differential equations with Caputo-Katugampola fractional derivative. 


\section{References}

[1] H . J. Haubold, A. M. Mathai, and R. K. Saxena, Mittag-Leffler Functions and Their Applications, Jour of App Math Volume 2011, Article ID 298628, 51 pages.

[2] Almeida, R., Malinowska, A. B., Odzijewicz, T., "Fractional Differential Equations With Dependence on the Caputo-Katugampola Derivative," J. Comput. Nonlinear Dynam, Vol. 11, pp. 061017 (2016).

[3] Katugampola, U. N., " Existence and uniqueness results for a class of generalized fractional differential equations, preprint arXiv: 1411.5229 .

[4] Katugampola, U. N., "A new approach to generalized fractional derivatives," Bull. Math. Anal. Appl., Vol. 6, pp. 1-15 (2014).

[5] Hilfer, R., "Applications of Fractional Calculus in Physics, "World Scientific, Singapore (2000).

[6] Tarasov, V. E., " Fractional Dynamics: Application of Fractional Calculus to Dynamics of Particles, Fields and Media "Springer, Heidelberg; Higher Education Press, Beijing (2010).

[7] Kilbas, A. A., Srivastava, H. M., Trujillo, J. J., " Theory and Applications of Fractional Differential Equations " Elsevier Science B.V., Amsterdam (2006).

[8] Miller, K. S., Ross, B., " An Introduction to the Fractional Calculus and Differential Equations "John Wiley, New York (1993).

[9] Bai, Y., Kong, H., "Existence of solutions for nonlinear Caputo-Hadamard fractional differential equations via the method of upper and lower solutions," J. Nonlinear Sci. Appl., Vol. 10, pp. 5744-5752 (2017).

[10] Guo, T. L., Zhang, K., "Impulsive fractional partial differential equations," Applied Mathematics and Computation, Vol. 257, pp. 581-590 (2015).

[11] Zhang, X., "On impulsive partial differential equations with Caputo-Hadamard fractional derivatives," Advances in Difference Equations, Vol. 2016, pp. 281 (2016).

[12] Cernea, A., "On the Solutions of a Class of Fractional Hyperbolic Integro-Differential Inclusions," International Journal of Analysis and Applications, Vol. 17, pp. 904-916 (2019).

[13] Erdélyi, A. and Kober, H., "Some remarks on Hankel transforms," Q. J. Math., Vol. 11, pp. 212-221 (1940).

[14] Katugampola, U. N., "A new approach to generalized fractional derivative," Bull. Math. Anal. Appl., Vol. 6, pp. 1-15 (2014). 